\title{
A visão do graduando de enfermagem quanto sua atuação no mercado de trabalho do Sistema Único de Saúde
}

\author{
The view of the nursing graduate as to his performance in the labor market of the Unified \\ Health System
}

\section{Lá visión del graduado en enfermería sobre su desempeño en el mercado de trabajo del Sistema Único de Salud}

Hulda Alves de Araújo Tenório ${ }^{1 *}$, Divanise Suruagy Correia ${ }^{1}$, Alda Galdino dos Santos², Emilly Souza Marques ${ }^{2}$, Esvaldo dos Santos Silva ${ }^{3}$, Douglas de Oliveira Subrinho4.

\section{RESUMO}

Objetivo: Analisar a visão do graduando de enfermagem quanto sua atuação no mercado de trabalho do Sistema Único de Saúde. Métodos: Trata-se de um estudo analítico, transversal e de abordagem quantitativa, realizado com 150 graduandos de enfermagem de uma faculdade particular em Alagoas. Os dados foram coletados e tabulados em planilha eletrônica Microsoft Exce目 e analisados em software aplicativo Statistical Package for Social Science - SPSS®, versão 20. Resultados: Os estudantes apresentaram a idade média de 27 anos, 82\% deles positivam satisfação quanto sua formação acadêmica. Destaca-se o desejo em 96,7\% dos discentes em experienciar uma maior vivência de Ensino durante a graduação no SUS, com 57,3\% afirmando o interesse em uma disciplina eletiva de conteúdo prático de saúde coletiva e a Estratégia Saúde da Família. O odds ratio de 3,69 mostra que o estudante que possuía alguma experiência de ensino em disciplinas no SUS tem 3,69 vezes mais chances de apresentar capacidade de realizar atividades junto a outros profissionais da saúde que o estudante que não obteve esta experiência no SUS. Conclusão: Assim, a inclusão dos estudantes de enfermagem nos campos de atuação de Enfermeiros no SUS, parece proporcionar, ao futuro egresso, uma melhor perspectiva de atuação no mercado de trabalho.

Palavras-chave: Mercado de trabalho, Educação em enfermagem, Sistema único de saúde.

\begin{abstract}
Objective: To analyze the view of nursing graduates as to his performance in the work Market of the Unified Health system. Methods: This is an analytical and cross-sectional study and has a quantitative approach, carried out with 150 nursing undergraduates from a private college in Alagoas. Data were collected and tabulated in a Microsoft Excel $\AA$ spreadsheet and analyzed in Statistical Package for Social Science - SPSS $\AA$, version 20 software. Results: The students presented the average age of 27 years, $82 \%$ of them positivam satisfaction as their academic formation. It is worth mentioning the desire of $96.7 \%$ of the students to experience a higher education experience during the SUS graduation, with $57.3 \%$ affirming the interest in an elective subject of practical content of collective health and the Family Health Strategy. The odds ratio of 3.69 shows that the student who had some teaching experience in disciplines in the SUS is 3.69 times more likely to be able to perform activities with other health professionals than the student who did not obtain this experience in the SUS. Conclusion: Thus, the inclusion of nursing students in the fields of Nursing in the SUS, seems to provide, to the future egress, a better perspective of work in the labor Market.
\end{abstract}

Key words: Labor market, Nursing education, Single health system.

\footnotetext{
${ }^{1}$ Universidade Federal de Alagoas da Faculdade de Medicina (UFAL), Maceió - AL. *E-mail: hulda.alves@outlook.com

${ }^{2}$ Mestre em Enfermagem pela Esenfar - UFAL.

${ }^{3}$ Faculdade Estácio de Alagoas.

${ }^{4}$ Centro Universitário Cesmac - Maceió - AL.
}

SUBMETIDO EM: 5/2019

ACEITO EM: 6/2019

PUBLICADO EM: $8 / 2019$ 


\section{RESUMEN}

Objetivo: Analizar la visión de los estudiantes de enfermería sobre su desempeño en el mercado de trabajo del Sistema Único de Salud. Métodos: Se trata de un enfoque analítico, transversal y cuantitativo, realizado con 150 estudiantes de enfermería de un colegio privado en Alagoas. Los datos se recopilaron y tabularon en una hoja de cálculo de Microsoft Excel® y se analizaron en Statistical Package for Social Science (SPSS), versión 20. Resultados: Los estudiantes presentaron la edad promedio de 27 años, $82 \%$ de ellos con satisfacción positiva com su entrenaniento académicos. Cabe mencionar el deseo del $96.7 \%$ de los estudiantes de experimentar una mayor experiencia de educacion durante la graduación del SUS, con un $57.3 \%$ que afirma el interés en un tema electivo de contenido práctico de salud colectiva y la Estrategia de Salud Familiar. El odds ratio de 3.69 muestra que el estudiante que tuvo alguna experiencia docente en disciplinas en el SUS tiene 3.69 veces más probabilidades de poder realizar actividades con otros profesionales de la salud que el estudiante que no obtuvo esta experiencia en el SUS. Conclusión: Por lo tanto, la inclusión de los estudiantes de enfermería en los campos de la Enfermería en el SUS, parece proporcionar, para el futuro egreso, una mejor perspectiva del atuacion en el mercado de trabajo.

Palabras clave: Mercado de trabajo, Educación en enfermería, Sistema único de salud.

\section{INTRODUÇÃO}

O Sistema Único de Saúde (SUS) criado a partir da Constituição Federal de 1988 (CF/1988) teve como marco primordial proporcionar um melhor acesso à saúde pela população brasileira. Tendo como pilares de sustentação seus princípios e diretrizes, este sistema garantiu o direito à saúde tendo como fundamento os princípios da integralidade, equidade e universalidade. Ademais, a consolidação do SUS estimulou o crescimento de diversos segmentos industriais e de serviços voltados ao suprimento das necessidades que englobam o contexto saúde (DEDECCA CS e TROVAO CJBM, 2013).

Em um estudo transversal cuja população alvo foi constituída por 1,8 milhões de profissionais de enfermagem, com registro ativo no Conselho Federal de Enfermagem (COFEN), com objetivo de destacar as modalidades institucionais em que as equipes de enfermagem desenvolviam suas atividades percebeu-se que $56,6 \%$ desses profissionais trabalhavam em hospitais sejam eles públicos ou privados, $18,1 \%$ atuavam em Unidades Básicas de Saúde (UBS) e outros serviços de saúde similares como Centro de Atenção Psicossocial (CAPS) e Núcleo de Apoio a Saúde da Família (NASF), 6,1\% atuavam em ambulatórios que incluíram clínicas, policlínicas, unidades mistas e outros serviços sem internação e $0,1 \%$ em unidades de diagnóstico e terapia (MACHADO MH et al., 2016).

Os resultados acima evidenciam a magnitude das áreas de atuação dos recursos humanos em saúde, em destaque os de enfermagem, sendo identificada uma crescente absorção desta categoria nas diferentes esferas de governo e níveis de complexidade. Porém, para atender às novas determinações da realidade econômica, social e política do país, esse mercado de trabalho passa a exigir profissionais com formação generalista, capazes de atuar em diferentes níveis de atenção à saúde (BARROS NJM et al., 2014).

A formação desses profissionais deve seguir os pressupostos da reforma curricular dos cursos de graduação. Tal reforma emergiu da necessidade de reorientar os currículos pedagógicos, a partir dos desafios educacionais colocados pela globalização onde o conhecimento incorpora um papel fundamental (MARTINI JG et al., 2017).

Neste sentido, foi aprovada a Resolução CNE/CES no 3 de 07 de novembro de 2001, que definiu as Diretrizes Curriculares Nacionais para o Curso de Graduação em Enfermagem (DCN/ENF). As DCN/ENF, atualmente em vigor, estimulam as mudanças nas fragilidades presentes na prática assistencial onde a atuação profissional dos Enfermeiros se volta para ações tecnicistas e de teor curativo, com base em um modelo de formação reducionista e previamente formulado (MARTINI JG et al., 2017; FERNANDES JD e REBOUCAS LC, 2013).

Esse modelo de formação estimula experiências práticas no ensino, dentro de um cenário real de saúde, formando profissionais mais críticos, criativos e resolutivos. Tais pressupostos indicam que os desafios serão 
múltiplos tornando-se de fundamental a realização de estudos que aprofundem as imprecisões teóricas, salientando as superações das dificuldades, sendo possível em transpor orientações propostas nas DCN/ENF para os cenários do processo de formação (FERNANDES JD e REBOUCAS LC, 2013). Assim, surge a seguinte questão norteadora da pesquisa: qual a visão do graduando de enfermagem quanto sua atuação no mercado de trabalho do Sistema Único de Saúde?

A hipótese da pesquisa é que os estudantes de Enfermagem estejam sendo formados com vistas positivas de sua atuação no mercado de trabalho no SUS, observados a partir do desenvolvimento de habilidades e competências desempenhadas durante a graduação para o desenvolvimento de uma assistência diante da realidade desse sistema (COSTA SM et al., 2012). O estudo teve como objetivo geral analisar a visão do graduando de enfermagem quanto sua atuação no mercado de trabalho do Sistema Único de Saúde.

Esse estudo se justificou, pois, o modelo de atenção sugerido pelo SUS implica no desafio de redirecionar as práticas de prestação de serviços, além da formação de profissionais de saúde para um olhar direcionado as necessidades individuais e coletivas da população.

Sendo um dos objetivos/atribuições do SUS a participação na formação dos recursos humanos em saúde, se faz necessário que as escolas estejam amparadas em políticas intersetoriais que reflitam práticas de formação reais, baseadas no atual sistema de saúde que norteia seu cuidar (FERNANDES JD e REBOUCAS LC, 2013).

\section{MÉTODOS}

Trata-se de um estudo analítico, transversal e de abordagem quantitativa realizado em 2016 com graduando de enfermagem de uma faculdade particular em Alagoas. Os discentes foram distribuídos entre os 10 períodos do curso de graduação em enfermagem para garantir a participação dos vários níveis de desenvolvimento acadêmico que compõem o curso de enfermagem. A amostra foi composta por 15 estudantes para cada período resultando em um total de 150 participante, cuja amostragem foi não probabilista e por conveniência. Os graduandos responderam a um questionário produzido já reconhecido através da literatura nacional e internacional (SANTOS, 2009) adaptado ao curso de Enfermagem, composto por 26 perguntas semiestruturadas, composto por 05 partes: perfil sociodemográfico dos pesquisados, impressões dos sujeitos em relação ao curso, processo de ensino e aprendizagem, mercado de trabalho em enfermagem e saúde coletiva (SUS e ESF). O tempo médio para preenchimento do instrumento foi de 15 minutos.

Os dados coletados na pesquisa foram tabulados em planilha eletrônica Microsoft Exceß e analisados em software aplicativo Statistical Package for Social Science - SPSS $®$, versão 20. As análises foram realizadas com base no tipo de dado (nominal, ordinal, contínuos e discretos) e para a análise estatística descritiva foram calculadas: frequência, média, desvio-padrão, erro padrão da média e Intervalo de Confiança de $95 \%$. Para a análise inferencial foram utilizados os teste Qui-quadrado, seguido do Teste Exato de Fisher em casos dos valores esperados menores de 5 .

Para comparação de médias entre os grupos foi utilizado o teste t de student de amostras independentes. Para todas as análises as diferenças entre os grupos foram consideradas significativas com valores de $p<$ 0,05 . Foram consideradas as diferenças detectadas das variáveis entre os grupos que se percebiam capazes em realizar atividades relacionadas à sua atuação no SUS. O projeto foi aprovado pelo CEP da Faculdade Estácio de Alagoas com processo número 1.380.775 e CAAE: 46912515.4 .0000 .5012 em 23 de dezembro de 2015.

\section{RESULTADOS}

A amostra foi composta por 150 alunos de enfermagem e apresentou a idade média de 27 anos (desvio padrão $\pm 7,181$ ), sendo $88 \%$ com predominância do sexo feminino, $46 \%$ natural do interior de Alagoas. Em relação às expectativas ao curso $76,6 \%$ deles afirmaram estarem supridos em sua formação em Enfermagem, e ainda $82 \%$ positivam satisfação quanto esta formação para o mercado de trabalho no SUS (Tabela 2). 
Tabela 2 - Frequência de Expectativas em Relação ao Curso e sobre a Capacidade em Preparar para o Mercado de Trabalho. Maceió - AL, 2016.

\begin{tabular}{|c|c|c|}
\hline & \multicolumn{2}{|c|}{0 curso atende às suas expectativas profissionais } \\
\hline & $\mathbf{N}$ & $(\%)$ \\
\hline Sim & 115 & 76,6 \\
\hline Não & 1 & 0,7 \\
\hline \multirow[t]{3}{*}{ Em parte } & 34 & 22,7 \\
\hline & \multicolumn{2}{|c|}{ Capacidade do curso em preparar para o mercado de trabalho } \\
\hline & $\bar{N}$ & $(\%)$ \\
\hline Sim & 123 & 82 \\
\hline Não & 10 & 6,7 \\
\hline Não sei & 17 & 11,3 \\
\hline
\end{tabular}

Fonte: Produzido pela autora, 2016.

Contudo, destaca-se o desejo em 96,7\% dos discentes em experienciar uma maior vivência de Ensino durante a graduação no SUS, com 57,3\% afirmando o interesse em uma disciplina eletiva com conteúdo prático de saúde coletiva e a Estratégia Saúde da Família (ESF). Além disso, 68\% já haviam vivenciado experiências de ensino em disciplinas no SUS (Tabela 3).

Tabela 3 - Frequência em ser favorável a maior vivência no SUS, em cursar disciplina eletiva de Saúde Coletiva e ter vivenciado experiência de Ensino no SUS. Maceió - AL, 2016.

\begin{tabular}{|c|c|c|c|}
\hline & \multicolumn{3}{|c|}{ Favorável a proposta do curso em maior vivência no SUS } \\
\hline & & $\mathbf{N}$ & $(\%)$ \\
\hline Sim & & 145 & 96,7 \\
\hline \multirow[t]{3}{*}{ Não } & & 5 & 3,3 \\
\hline & \multicolumn{3}{|c|}{ Cursar a Disciplina de Saúde Coletiva em caso de Disciplina Eletiva } \\
\hline & \multicolumn{2}{|l|}{$\mathbf{N}$} & $(\%)$ \\
\hline Sim & \multicolumn{2}{|l|}{86} & 57,3 \\
\hline \multirow[t]{3}{*}{ Não } & 64 & & 42,7 \\
\hline & \multicolumn{3}{|c|}{ Experiência de ensino no SUS } \\
\hline & \multicolumn{2}{|l|}{$\mathbf{N}$} & (\%) \\
\hline Sim & \multicolumn{2}{|l|}{102} & 68 \\
\hline Não & \multicolumn{2}{|l|}{48} & 32 \\
\hline
\end{tabular}

Fonte: Produzido pela autora, 2016.

Sobre à capacidade de realizar educação em saúde para grupos populacionais, os pesquisados que demonstraram maior aptidão estavam entre aqueles que responderam também possuir experiência de ensino em disciplinas no SUS (odds $=3,8 ; p=0,001$,).

Os alunos que referiram sentir-se preparado para atuar no SUS, apresentaram uma associação estatisticamente significativa para a capacidade de realizar educação em saúde para grupos populacionais (odd $=3,92 ; p=0,029$ ).

Estudantes que demonstraram interessem em realizar disciplinas que envolvem conteúdos de saúde coletiva no SUS apresentaram resultados de associação estatística significativa com a capacidade de realizar educação em saúde para grupos populacionais (odds =3,29; $p=0,02$ ) (Tabela 4). 
Tabela 4 - Associação entre Percepções sobre Mercado de Trabalho em Enfermagem e Saúde e Capacidade de Realizar Educação em Saúde. Maceió -AL, 2016. Capacidade de realizar educação em saúde para
grupos populacionais

\begin{tabular}{|c|c|c|c|c|c|c|c|}
\hline & \multicolumn{2}{|c|}{ Sim } & \multicolumn{2}{|c|}{ Não } & \multirow{2}{*}{ P Valor } & \multirow{2}{*}{ OR } & \multirow{2}{*}{ IC 95\% } \\
\hline & $\mathrm{N}$ & $\%$ & $\mathrm{~N}$ & $\%$ & & & \\
\hline \multicolumn{8}{|c|}{$\begin{array}{l}\text { O curso de Enfermagem da Instituição de } \\
\text { Ensino Superior (IES) está capacitando para a } \\
\text { inserção no atual mercado de trabalho }\end{array}$} \\
\hline $\operatorname{Sim}$ & 96 & 64 & 27 & 18 & & & \\
\hline Não & 9 & 6 & 1 & 0,7 & $0,318^{*}$ & - & - \\
\hline Não sei & 11 & 7,3 & 6 & 4 & & & \\
\hline \multicolumn{8}{|c|}{$\begin{array}{l}\text { O mercado de trabalho está propício aos } \\
\text { futuros Enfermeiros assistenciais }\end{array}$} \\
\hline Sim & 82 & 54,7 & 22 & 14,7 & & & \\
\hline Não & 23 & 15,3 & 9 & 6,0 & $0,669^{*}$ & - & - \\
\hline Não sei & 11 & 7,9 & 3 & 2,0 & & & \\
\hline \multicolumn{8}{|c|}{ Onde gostaria de exercer a profissão } \\
\hline Interior de Alagoas & 46 & 30,7 & 13 & 8,7 & & & \\
\hline Maceió & 39 & 26,0 & 9 & 6,0 & & & \\
\hline Grandes centros & 14 & 9,3 & 7 & 4,7 & $0,581^{*}$ & - & - \\
\hline Não sei & 9 & 6,0 & 4 & 2,7 & & & \\
\hline Outro & 8 & 5,3 & 1 & 0,7 & & & \\
\hline \multicolumn{8}{|l|}{ Pretende especializar } \\
\hline $\operatorname{Sim}$ & 115 & 76,7 & 34 & 22,7 & $1,00^{*}$ & 0,77 & $0,70-0,84$ \\
\hline Não & 1 & 0,7 & 0 & 0 & & & \\
\hline \multicolumn{8}{|c|}{ Possui alguma experiência de ensino no SUS } \\
\hline Sim & 87 & 58,0 & 15 & 10 & 0,001 & 3,8 & $1,71-8,43$ \\
\hline Não & 29 & 19,3 & 19 & 12,7 & & & \\
\hline \multicolumn{8}{|c|}{ Sente-se preparado para atuar no SUS } \\
\hline Sim & 110 & 73,3 & 28 & 18,7 & $0,029^{\star}$ & 3,92 & $1,17-13,11$ \\
\hline Não & 6 & 4,0 & 6 & 4,0 & & & \\
\hline \multicolumn{8}{|c|}{$\begin{array}{l}\text { Faria as disciplinas que envolvem o conteúdo } \\
\text { da Saúde Coletiva se optativas. }\end{array}$} \\
\hline Sim & 110 & 73,3 & 28 & 18,7 & 0,02 & 3,92 & $1,17-13,11$ \\
\hline Não & 6 & 4 & 6 & 4 & & & \\
\hline
\end{tabular}

${ }^{*}$ Realizado teste exato de Fisher considerando $p<0,05$.

Fonte: produzido pela autora, 2016.

Quando foram questionados sobre a capacidade de realizar atividades preventivas e de promoção em saúde juntamente com profissionais de outras áreas da saúde (atividades multiprofissionais), os estudantes demonstraram mais esta capacidade no grupo que respondeu também ter possuído experiência de ensino em disciplinas no SUS, embasado na presença de diferença estatística $(p=0,018)$.

O odds ratio de 3,69 mostra que o estudante que possuía alguma experiência de ensino em disciplinas no SUS tem razão de chances em 3,69 vezes maior de apresentar capacidade de realizar atividades preventivas e de promoção em saúde junto a outros profissionais da saúde que aquele estudante que não obteve esta experiência no SUS (Tabela 5). 
Tabela 5 - Associação entre às Atividades Preventivas e de Promoção em Saúde juntamente com outros Profissionais de Saúde. Maceió - AL, 2016.

\begin{tabular}{|c|c|c|c|c|c|c|c|}
\hline & \multicolumn{7}{|c|}{$\begin{array}{l}\text { Capacidade de realizar atividades de prevenção e } \\
\text { promoção em Saúde na equipe multidisciplinar. }\end{array}$} \\
\hline & \multicolumn{2}{|c|}{ Sim } & \multicolumn{2}{|c|}{ Não } & \multirow[t]{2}{*}{$\mathrm{P}$ valor } & \multirow[t]{2}{*}{ OR } & \multirow[t]{2}{*}{ IC 95\% } \\
\hline & $\mathrm{N}$ & $\%$ & $\mathrm{~N}$ & $\%$ & & & \\
\hline \multicolumn{8}{|l|}{ Expectativa de trabalho depois da graduação } \\
\hline Setor público & 31 & 20,7 & 6 & 4 & & & \\
\hline Setor privado & 2 & 1,3 & 0 & 0,0 & & & \\
\hline Setor público e privado & 96 & 64 & 8 & 5,3 & & & \\
\hline Magistério & 3 & 2,0 & 1 & 0,7 & 0,404 & - & - \\
\hline Administração dos serviços de saúde & 1 & 0,7 & 0 & 0,0 & & & \\
\hline Pesquisa & 2 & 1,3 & 0 & 0,0 & & & \\
\hline \multicolumn{8}{|l|}{$\begin{array}{l}\text { O curso de Enfermagem da IES está } \\
\text { capacitando para a inserção no atual } \\
\text { mercado de trabalho }\end{array}$} \\
\hline Sim & 109 & 72,7 & 14 & 9,3 & & & \\
\hline Não & 10 & 6,7 & 0 & 0,0 & 0,064 & - & - \\
\hline Não sei & 16 & 10,7 & 0 & 0,0 & & & \\
\hline \multicolumn{8}{|l|}{$\begin{array}{l}\text { O mercado de trabalho está propício aos } \\
\text { futuros Enfermeiros assistenciais }\end{array}$} \\
\hline Sim & 91 & 60,7 & 13 & 8,7 & & & \\
\hline Não & 31 & 20,7 & 1 & 0,7 & 0,373 & - & - \\
\hline Não sei & 13 & 8,7 & 1 & 0,7 & & & \\
\hline \multicolumn{8}{|l|}{ Onde gostaria de exercer a profissão } \\
\hline Interior de Alagoas & 54 & 36 & 5 & 3,3 & & & \\
\hline Maceió & 43 & 28,7 & 5 & 3,3 & & & \\
\hline Grandes centros & 17 & 11,3 & 4 & 2,7 & 0,622 & - & - \\
\hline Não sei & 12 & 8 & 1 & 0,7 & & & \\
\hline Outro & 9 & 6 & 0 & 0,0 & & & \\
\hline \multicolumn{8}{|l|}{ Pretende especializar } \\
\hline Sim & 134 & 89,3 & 15 & 10 & & & \\
\hline Não & 1 & 0,7 & 0 & 0,0 & 0,900 & 0,899 & $0,852-0,949$ \\
\hline \multicolumn{8}{|l|}{ Possui alguma experiência de ensino no SUS } \\
\hline $\operatorname{Sim}$ & 96 & 64 & 6 & 4 & & & \\
\hline Não & 39 & 26 & 9 & 6 & 0,018 & 3,69 & $1,23-11,07$ \\
\hline \multicolumn{8}{|l|}{ Sente-se preparado para atuar no SUS } \\
\hline Sim & 104 & 69,3 & 9 & 6 & & & \\
\hline Não & 31 & 20,7 & 6 & 4 & 0,129 & 2,23 & $0,73-6,77$ \\
\hline \multicolumn{8}{|l|}{$\begin{array}{l}\text { Faria as disciplinas que envolvem o conteúdo } \\
\text { da Saúde Coletiva se optativas. }\end{array}$} \\
\hline Sim & 126 & 84 & 12 & 8 & & & \\
\hline Não & 9 & 6 & 3 & 2 & 0,103 & 3,50 & $0,83-14,69$ \\
\hline
\end{tabular}

${ }^{\star}$ Realizado teste exato de Fisher considerando $p<0,05$. Fonte: produzido pela autora, 2016.

\section{DISCUSSÃO}

No estudo em questão, 88\% (Tabela 1) dos sujeitos são do sexo feminino, confirmando a tendência ainda feminina que a equipe de enfermagem carrega, marcada pela feminização da profissão com porcentagens relativas a 90\% (MACHADO $\mathrm{MH}$, et al., 2012). 
Contudo, dados apontam para um crescimento constante, na década de 90, de homens neste cenário, que se intensificou a partir da reforma universitária em 1968 e possibilitou a inserção do sexo masculino no curso de enfermagem por aprovação no vestibular e não apenas por vocação ou por condutas consideradas adequadas (COSTA e FREITAS, 2017).

Apesar da crescente ascensão masculina na profissão, conota-se em tempos atuais, um preconceito histórico oriundo das diferenças de gênero e seus papéis sociais. Em estudo realizado com moradores da cidade de Itajubá-MG atendidos por enfermeiros do sexo masculino, foram relatados a resistência e preconceito por parte da população atendida, sendo esse comportamento predominante. Constatou-se que apesar da "aceitação" e da "conquista" dos homens e sua profissionalização na enfermagem, aspectos como o constrangimento de usuárias mulheres diante de alguns conteúdos e de alguns procedimentos quando realizados por profissionais homens, foram referidos como embaraçosos (VITORINO DFP et al., 2012).

Características inerentes ao perfil sociodemográfico dos estudantes de Enfermagem, podem contribuir para a ocorrência do estresse durante sua formação. A ampliação de diretrizes para uma política de assistência estudantil baseada no princípio da autonomia universitária, contribuiu significativamente para a inserção de alunos nas graduações. O Programa de Apoio a Planos de Reestruturação e Expansão das Universidades Federais - REUNI, o programa Universidade para todos - PROUNI e o programa de financiamento estudantil -FIES- protagonizaram esta ampliação carregando em si uma estratégia expansionista para ampliar o espaço social da educação superior (BLEICHER T e OLIVEIRA RCN, 2016; CORRÊA LZM, et al., 2014).

Essa expansão deu-se também para alunos que residem em cidades menores, favorecendo a interiorização dos ensinos superiores, com maior oportunidade de formação aos munícipes de cidades menos favorecidas. Contudo, quando aspectos sociodemográficos são confrontados em estudos, percebe-se que as distâncias entre a sede das Instituições de Ensino Superior (IES) e o local de residência dos discentes interferem na qualidade da formação acadêmica. Em estudo realizado com 130 acadêmicos de enfermagem do $1^{\circ}$ aos $8^{\circ}$ períodos do curso houve destaque, como segundo maior fator estressante, o "ambiente" com média de 1,89 ( $\mathrm{dp}=0,81)$. Esses autores apontaram que o deslocamento dos estudantes de suas residências para as universidades, locais das aulas práticas e campos de estágios, bem como as condições do transporte público utilizado, foram referidos como estressores (BUBLITZ S, et al., 2012).

A qualidade de vida dos estudantes de enfermagem durante a graduação foi avaliada em estudo e constataram que $64,6 \%$ desses alunos moravam em municípios distantes e destacaram que $22,2 \%$ afirmaram a presença de fadiga e estresse, tendo como desencadeadores a distância entre a casa e a faculdade, bem como as condições de transporte (MOURA IH, et al., 2016). Tal fato assemelha-se a realidade dos sujeitos da atual pesquisa com $69 \%$ de discentes oriundos de cidades do interior de Alagoas, podendo tal fator colaborar com aspectos negativos no âmbito do ensino e da aprendizagem.

Outra questão apontada nesse estudo, foi à satisfação dos acadêmicos quanto ao curso de enfermagem, com $76,6 \%$ (Tabela 2) de aprovação, à instituição estudada vem contribuindo para uma boa experiência acadêmica. Vale ressaltar que a pesquisa em questão evidenciou apenas a visão do discente quanto sua formação, não adentrando a aspectos que exemplifique e que resgatem a prática da intersecção entre a formação e o mercado de trabalho, tal afirmativa pode justificar o total de 11,3\% (Tabela 2) dos alunos não obterem respostas concretas sobre sua qualificação.

Quanto aos aspetos relacionados à satisfação acadêmica para formação ao mercado de trabalho do SUS, $82 \%$ dos alunos (Tabela 2) apontaram que a instituição de ensino contribuía adequadamente neste quesito. No que concerne a esta prática, a aderência à formação profissional na perspectiva do SUS vem levando a alterações no modelo pedagógico acadêmico, buscando reorientá-lo para as reais necessidades da sociedade. Em estudo em que discursam 13 discentes distribuídos em quatro IES públicas e particulares foi possível identificar a presença da articulação do processo de formação do enfermeiro com o SUS a partir de práticas de ensino em vários cenários de aprendizagem e dos conteúdos curriculares que discutem os princípios e diretrizes deste sistema (FERNANDES JD, et al., 2013). 
Uma forma de aproximar os futuros trabalhadores do SUS ao seu contexto real é através de vivências neste cenário. A falta de profissionais que atuem adequadamente no SUS, a partir de uma formação voltada para esse sistema, tem sido um fator comprometedor para a gestão e para a melhoria da qualidade da assistência ofertada. Tal fato emerge e demanda um constante movimento de mudança no sentido de enfrentar essas dificuldades (DIAS IMAV, et al., 2016).

Quanto a este aspecto $96,7 \%$ dos pesquisados (Tabela 3) são favoráveis a esta ampliação, na verdade, tais mudanças já vêm sendo incorporadas pelos cursos de graduação a partir das Diretrizes Curriculares de Ensino (DCN) desde 2001.

As Diretrizes Nacionais para o Curso de Graduação em Enfermagem (DCN/ENF) surgiram na resolução no 03 de 7/11/2001 e apontou a necessidade de articulações intersetoriais entre a educação e saúde, trazendo indicadores de mudança no processo de formação do enfermeiro. O perfil do egresso/profissionais passou a ter como objetivo uma assistência generalista, crítica e reflexiva com ênfase na promoção, prevenção, recuperação e reabilitação da saúde (FERNANDES JD, et al., 2013).

Em um estudo bibliográfico recente foi possível perceber que mesmo após 16 anos dos direcionamentos apontados pela DCN/ENF o índice de aderência ainda foi considerado baixo, revelando a necessidade das IES buscarem maior vinculação entre a adoção das bases epistemológicas presentes nas diretrizes e as propostas contidas nos projetos político pedagógico. Ainda, em estudo em que foram agrupados os depoimentos de discentes sobre a articulação das DCN no curso de Enfermagem, alguns revelaram não perceberem esse processo, negando a existência de articulação, o que levou a uma insegurança dos discentes quanto ao seu fazer (MARTINI JG, et al., 2017; FERNANDES JD, et al., 2013).

O aumento da carga horária disponibilizada para disciplinas de caráter prático promove aos sujeitos mais segurança e autoconfiança, sendo fatores que contribuem para o reforço da identidade profissional através do aumento da experiência e conhecimentos clínicos (HIRSCH CD, et al., 2015). No estudo em questão 36\% dos alunos (Tabela 3) ainda não vivenciaram nenhuma situação de prática no SUS e 57,3\% são favoráveis a cursar uma disciplina eletiva de saúde coletiva que abordem o contexto do SUS e da ESF.

Ao analisar a Projeto Político Pedagógico da IES estudada verificou-se que os alunos iniciam suas atividades de campo nos cenários do sistema de saúde a partir do 4º período do curso. No ano de 2016 houve uma alteração na matriz curricular do curso, onde a disciplina de Saúde da Família, com enfoque ao SUS, foi incorporada a matriz pedagógica dos alunos do $3^{\circ}$ período do curso, porém de acordo com a ementa da disciplina, a mesma se reporta apenas aos aspectos teóricos e de aplicações em sala de aula. $O$ fato de não estarem vivenciado práticas no SUS, desde os primeiros períodos da graduação como orienta as DCN/ENF é um dado preocupante, visto existirem evidências recentes de uma tendência estudantil à poucas atividades extracurriculares, o que limita consideravelmente sua experiência de formação.

Em uma pesquisa que avaliou às atividades desenvolvidas pelos estudantes de enfermagem no último período do curso, $15 \%$ dos alunos haviam desenvolvido atividades de extensão, $9 \%$ atividades de monitoria e atividades de extensão, $8 \%$ de monitoria e $14 \%$ deles desenvolvido todas as atividades ofertadas dentro da graduação, porém um montante de $42 \%$, não havia desenvolvido atividade nenhuma, limitando-se apenas aos conteúdos propostos pelo curso (OLIVEIRA LB, et al., 2015).

Ainda, em outro estudo recente realizado por Bublitz e colaboradores (2015), mostrou que $71,7 \%$ dos alunos do primeiro ao último semestre do curso de enfermagem de faculdades públicas e privadas, não participaram de estudos pesquisa, incluindo disciplinas eletivas. É importante destacar que os grupos de estudo e pesquisa proporcionam maior vínculo entre a teoria e a prática, além de incentivar a busca pelo cientificismo a partir das ações realizadas, pois aprofundam o conhecimento e a leitura sobre os conteúdos constituindo-se um diferencial para o futuro enfermeiro.

No que concerne a aspectos da formação voltados as principais habilidades que deverão ser desenvolvidas por Enfermeiros em sua atuação no SUS a educação em saúde é um eixo primordial, visto englobar práticas para atuar em todos os níveis de atenção à saúde. 
Nesse contexto, a educação em saúde surge como um instrumento importante para a promoção à saúde, pois é capaz de proporcionar o empoderamento, ou seja, a capacidade da comunidade ou dos indivíduos, se tornarem agentes ativos do processo saúde-doença, dispondo assim de conhecimentos e habilidades para protagonizar o contexto saúde. Isso se distancia de uma prática de ensino verticalizada, mas de uma troca de conteúdos e conhecimentos onde as ações educativas possam promover a autonomia dos indivíduos, sem estarem regulados ou supervisionados por profissionais de saúde. A educação em saúde é capaz de desenvolver nos indivíduos autonomia diante de seu processo saúde-doença (OLIVEIRA MR, et al., 2015; OLIVEIRA LB, et al., 2015).

Neste estudo os alunos que vivenciaram experiências no SUS, apresentam 3,9 vezes mais chance de possuir capacidade de educar em saúde (Tabela 4), atribuindo-se a este dado a contribuição do exercício de tais práticas durante a graduação, o que os aproximam do serviço.

Sobre este quesito, alunos de Enfermagem de uma disciplina Educação Popular em Saúde (EPS) no primeiro ano do curso de graduação e que tiveram a experiência de capacitação dos Agentes Comunitários de Saúde (ACS) em EPS para o SUS, revelaram as potencialidades desta prática. Foram pontuados aprimoramento do conhecimento sobre trabalho dos agentes, além de seus saberes e práticas; houve aperfeiçoamento da pedagogia da problematização e de discussão dos problemas de saúde identificados pelos ACS e pelos alunos, sendo a EPS construída na perspectiva da promoção à saúde (AMARAL MCS, et al., 2014).

Outro aspecto observado na formação em enfermagem é a obrigatoriedade de atividades práticas diárias apenas nos últimos semestres da graduação compondo $20 \%$ da carga horária total do curso como preconiza a resolução no 441/13 do COFEN nos chamados estágios obrigatórios. Essa normativa pode engessar algumas IES a garantir esforços e bons campos apenas neste período. Em estudo que avaliou os estágios obrigatórios na visão do estudante de Enfermagem, foi possível constatar que tais atividades sozinhas, não foram capazes de proporcionar a segurança necessária para os alunos desenvolverem as atividades profissionais futuras, gerando ansiedade e medo, principalmente por estarem prestes a sair do mundo universitário (MARCHIORO D, et al., 2017).

Em pesquisa desenvolvida em um hospital escola com enfermeiros supervisores houve referência ao despreparo e a imaturidade com que os discentes vêm chegando aos últimos períodos. A falta de interesse e o despreparo teórico-prático do acadêmico para realizar o estágio vem comprometendo a realização deste. $A$ postura de desinteresse em aprender induz o enfermeiro a não investir na troca de informações com o acadêmico interferindo na troca de conhecimento, além do vínculo e da qualidade da assistência prestada (SOUZA DJ, et al., 2017).

No sentido das interações interpessoais que são apontados na atuação do contexto saúde, no estudo em questão, os participantes que responderam terem vivenciado práticas no SUS tinha 3,69 vezes mais chances de realizar atividades preventivas e de promoção em saúde juntamente com outros profissionais de outras áreas da saúde (Tabela 5), sendo aspectos da multidisciplinaridade e da interdisciplinaridade campos percorridos pelo SUS.

O trabalho interdisciplinar contempla, entre outros aspectos, a possibilidade de trabalho conjunto, que respeita as bases disciplinares específicas, buscando a partir delas, soluções compartilhadas para os problemas de saúde. É o encontro das diferentes disciplinas que perpassa pelos aspectos teóricos e práticos na construção de um novo saber, intervindo nas dimensões sociais, pedagógicas e de pesquisa (BISPO EPF, et al., 2014).

Sobre as práticas de extensão com enfoque nas atividades interdisciplinares, em projeto de extensão popular interdisciplinar desenvolvido na Universidade Federal da Paraíba (UFPB), os estudantes desenvolviam atividades de visitas domiciliares semanais, participavam de movimentos comunitários, das atividades junto à Unidade Básica de Saúde (UBS), de lutas sociais e de grupos de cuidado. Um dos pontos pleiteados pelo projeto foi o fato de levar os extensionistas a aprenderem a trabalhar em grupo através do respeito às diferentes maneiras de pensar e aos posicionamentos dos envolvidos; valorizando o tempo que 
cada sujeito tem para seu amadurecimento individual ou em sociedade; aprimorando valores como: "acolher o outro; a construir, construir junto, pensar junto, fazer junto" (LEITE MF, et al., 2014). Assim, os cursos de graduação em enfermagem devem apostar em currículos que atendam às necessidades de saúde da população no SUS, bem como as exigências do mercado de trabalho atual, intersectando o escopo da academia, aos objetivos dos serviços de saúde e às tendências do mercado de trabalho, a partir da construção de uma proposta pedagógica, pode garantir a empregabilidade dos discentes, além do desenvolvimento de práticas efetivas e resolutivas que atendam as demandas das comunidades (MAFFISSONI AL, et al., 2017).

\section{CONCLUSÃO}

A formação na área de saúde ainda é um desafio constante, visto que as alterações relacionadas ao mercado de trabalho definem o perfil do trabalhador que será absorvido nessa esfera. Nota-se, que os estudantes pesquisados esperam atuar no mercado de trabalho do SUS, porém as competências relacionadas a esta atuação foram efetivadas apenas quando inseridos dentro dos cenários que compõe 0 sistema. Graduandos que vivenciaram experiência de ensino em disciplinas no SUS ou que pretendem participar de disciplinas de saúde coletiva que abordem a temática do SUS e ESF possuíram uma maior capacidade de educar em saúde, desenvolver atividades de promoção à saúde na dimensão interdisciplinar. Portanto, parece importante inserir os estudantes de enfermagem nos campos de atuação de Enfermeiros que atuam no SUS, onde possam percorrer os cenários de atuação, tal prática poderá favorecer ao futuro egresso melhores perspectivas de atuação no mercado de trabalho, visto que no estudo em questão, não houve uma positividade uniforme dessa perspectiva.

\section{REFERÊNCIAS}

1. AMARAL MCS, et al. O ensino de Educação Popular em Saúde para o SUS: experiência de articulação entre graduandos de enfermagem e Agentes Comunitários de Saúde. Interface (Botucatu). 2014; 18 (supl. 2): $1547-1558$.

2. BARROS NJM et al. A formação do profissional enfermeiro e o mercado de trabalho na atualidade. Rev. Eletr. Gest. \& Saúd. 2014; 5: 176-193.

3. BISPO EPF et al. Interdisciplinaridade no ensino em saúde: o olhar do preceptor na Saúde da Família. Interface (Botucatu). 2014; 18 (49): 337-350.

4. BLEICHER T, OLIVEIRA RCN. Políticas de assistência estudantil em saúde nos institutos e universidades federais. Psicol. Esc. Educ. 2016; 20 (3): 543-549.

5. BUBLITZ $S$ et al. Estressores entre os acadêmicos de Enfermagem de uma Universidade Pública. Rev. Enferm. UERJ. 2012; 20 (esp.2): 739-745.

6. BUBLITZ $S$ et al. Perfil sociodemográfico e acadêmico de discentes de Enfermagem de quatro instituições Brasileiras. Rev. Gaúcha Enferm. 2015; 36 (1): 77-83.

7. CORRÊA LZM et al. Expansão dos cursos de graduação em enfermagem em Mato Grosso: implicações e desafios. Rev. Eletr. Enf. 2014; 16 (4): 744-753.

8. COSTA KS, FREITAS GF. Homens na enfermagem: formação acadêmica posterior à graduação e trajetória profissional. Rev. Enferm. UFPE. 2017; 11 (3): 1216-26.

9. COSTA SM, et al. Perceptions of dental students regarding dentistry the job Market and the publiche althcare system. Ciên. \& Saúde. Colet. 2012; 17 (5): 1285-1296.

10. DEDECCA CS, TROVAO CJBM. A força de trabalho no complexo da saúde: vantagens e desafios. Ciênc. Saúd. Colet. 2013; 18 (6): 1555-1567.

11. DIAS IMÁV et al. A tutoria no processo de ensino-aprendizagem no contexto da formação interprofissional em saúde. Saúde debate. 2016; 40 (111): 257-267.

12. FERNANDES JD et al. Aderência de cursos de graduação em enfermagem às diretrizes curriculares nacionais na perspectiva do sistema único de saúde. Esc. Anna Nery. 2013; 17 (1): 82-89.

13. FERNANDES JD, REBOUCAS LC. Uma década de Diretrizes Curriculares Nacionais para a Graduação em Enfermagem: avanços e desafios. Rev. Bras. Enferm. 2013; 66 (SPE): 95-101.

14. HIRSCH CD et al. Fatores preditores e associados à satisfação dos estudantes de enfermagem. Acta Paul. Enferm. 2015; 28 (6): 566-572.

15. LEITE MF et al. Extensão Popular na formação profissional em saúde para o SUS: refletindo uma experiência. Interface (Botucatu). 2014; 18 (supl. 2): 1569-1578. 
16. MAFFISSONI AL et al. VER-SUS Oeste Catarinense: vislumbrando um itinerário formativo em Enfermagem direcionado ao Sistema Único de Saúde. Rev. Enferm. UFPE. 2017; 11 (2): 758-64.

17. MARCHIORO D et al. Estágio curricular supervisionado: relato dos desafios encontrados pelos (as) estudantes. Arq. Cienc. Saúde UNIPAR. 2017; 21 (2): 119-122.

18. MARTINI JG, et al. Currículos de cursos de graduação em Enfermagem: revisão integrativa de literatura. Rev. Fund. Care. 2017; 9 (1): 265-272.

19. MACHADO MH, et al. Mercado de trabalho na Enfermagem: aspectos gerais. Enferm. Foco. $2016 ; 7$ (ESP): 35-62.

20. MACHADO MH, et al. Construindo o perfil da Enfermagem. Enferm. Foco. 2012; 3 (3): 119-122.

21. MOURA IH de et al. Qualidade de vida de estudantes de graduação em enfermagem. Rev. Gaúcha Enferm. $2016 ; 37$ (2): 552-91.

22. OLIVEIRA LB et al. Perfil sociodemográfico do discente do curso de graduação em enfermagem. Rev. Enferm. UFPI. 2015; 4 (1): 33-39.

23. OLIVEIRA MR et al. Concepção de graduandos de enfermagem sobre a prática e saúde em primeiros socorros. Rev. Rene. 2015; 16 (2): 150-158.

24. SOUZA DJ et al. Estágio curricular supervisionado sob a óptica dos enfermeiros supervisores. Rev. Enferm. Atenç Saúde. 2017; 6 (1): 39-51.

25. VITORINO DFP, et al. Percepção de moradores de uma cidade de minas gerais sobre o profissional de enfermagem do gênero masculino. Rev. Min. Enferm. Reme. 2012; 16 (4): 528-537. 